\title{
Optimisation of the High Efficiency Deep Grinding Process with Fuzzy Fitness Function and Constraints
}

\author{
P.M. Jones, A. Tiwari, R. Roy, J. Corbett \\ Department of Enterprise Integration \\ Cranfield University \\ Cranfield, Bedfordshire, UK, MK43 0AL \\ phil.jones@cranfield.ac.uk
}

\begin{abstract}
This paper describes the application of two multiobjective optimisation techniques to the high efficiency deep grinding process. The process is modelled using a fuzzy expert system. A description of how problem constraints can be represented within the fuzzy model is included. They form an objective representing the degree of infeasibility of the solution.

The paper concerns the high efficiency deep grinding process. The objectives are to simultaneously minimise the surface temperature and specific grinding energy.

The paper applies two multi objective evolutionary algorithms and reviews the results.
\end{abstract}

\section{INTRODUCTION}

This paper is concerned with finding the optimum parameters for efficient grinding without causing surface damage to the work piece. Specifically, it is to simultaneously minimise the surface temperature and grinding energy involved in the high efficiency deep grinding process (HEDG).

The paper consists of several sections. Section 2 describes the high efficiency deep grinding process. Section 3 is a concise overview of the main research in the area of fuzzy expert systems and multi objective optimisation. Section 4 outlines the proposed approach for this project. Section 5 gives results. These are then discussed in section 6 . Limitations and future work are in section 7 . The paper concludes in section 8 followed by references in section 9 .

\section{High EFFICIENCY DEEP GRINDING}

HEDG is a relatively new machining regime. It has been successfully applied in industry to achieve very high removal rates and good work piece surface integrity. Typical applications of HEDG include grinding of deep slots or grooves or simply removing a thick layer of material from the workpiece surface [6].

In traditional grinding, increases in depth of cut, feed rate and wheel speed cause increases in surface temperature. However it has been found that a dramatic increase in depth of cut and feed rate accompanied by an increase in wheel speed give lower than previously expected temperature. HEDG relative to conventional grinding, with respect to removal rate and temperature, is shown in figure 1.0. The wheel is in contact with the workpiece for less time. Thus reducing the amount of heat transferred to the finished product [12].

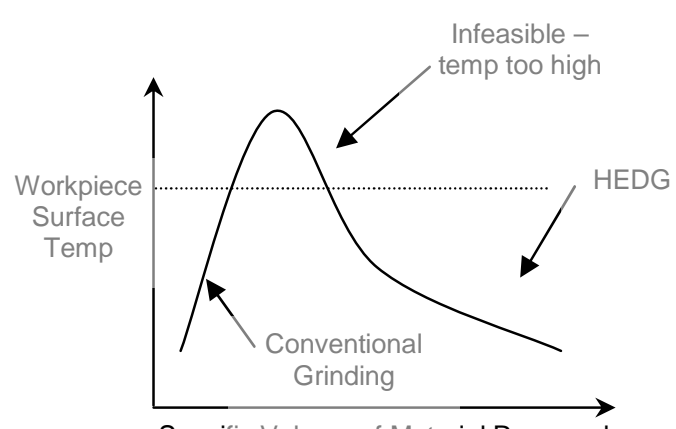

Figurecific Volume of Material Removed

The unique heat transfer conditions can cause thermal damage to the workpiece surface. The aim of this project is to choose the appropriate grinding parameters to realise efficient grinding without any thermal damage to the work piece.

The contact temperature can be calculated by predicting the various heat flux values. Rowe [9] gives the equation for total heat flux in the HEDG process as

$$
q_{t}=q_{w}+q_{s}+q_{c h}+q_{f}
$$

where $q_{t}$ is the total heat flux, $q_{w}$ is the heat flux into the work piece, $q_{s}$ is the heat flux into the wheel, $q_{c h}$ is the heat flux into the removed chips and $q_{f}$ is the heat flux into the coolant fluid. This is depicted in figure 2.0. 


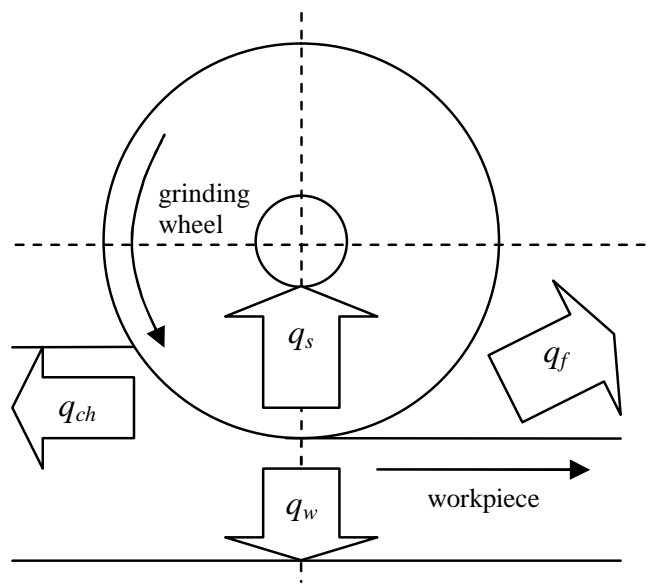

Figure 2.0 Schematic of Heat Flux in HEDG

The decision variables are the width of cut, feed rate, depth of cut and wheel speed. The objective is to minimise the surface temperature and the specific grinding energy. The specific grinding energy is equivalent to the power consumed per unit volume of material removed.

Analytical models have been put forward that predict the surface temperature of the work piece. These methods are derived from partial differential equations concerning the heat dissipation in and around the contact area [7].

The data used for this study relates to M50 hardened bearing steel. This is used in the aerospace and automotive industry. Specific surface finish is required. Any machine damage introduced during processing can cause rapid deterioration during use [11].

HEDG is a new process with some proposed theories and analytical models, the technician's expert opinion and a limited amount of empirical data. The challenge is to develop a model that can incorporate all three knowledge sources to describe the process and allow optimisation.

\section{LITERATURE REVIEW}

\section{A Fuzzy Expert Systems}

Lukasiewicz first developed a system of logic that extended the truth values to all real numbers in the range 0 to 1 . He used a number in this set to represent the possibility that a given statement was true or false. This research led to a formal inexact reasoning technique aptly named possibility theory.

In 1965, Zadeh extended the work on possibility theory into a formal system of mathematical logic. Zadeh also promoted fuzzy concepts and logic to the engineering and scientific community. These concepts are collectively referred to as fuzzy logic [3].

A Fuzzy Expert System (FES) comprises of three operations. First inputs are fuzzified. This converts numerical inputs into a set of degree of membership values. The second stage is inferencing here relevant rules are formed. Finally the consequent sets are defuzzified to give a crisp numeric output.

The knowledge required for a FES creation is in two distinct forms. Firstly, membership functions for all input and output variables. An example set of membership functions is shown in figure 2.0. Secondly, a rule base that relates input variables (antecedents) to output variables is required. This is simply a list of if...then... rules. For example

\section{If DOC is LARGE and FEED is HIGH then TEMP is V. HIGH \\ If WIDTH is LOW and WHLSPD is FAST then SGE is MEDIUM}

These two components are combined with the fuzzification, inferencing, and defuzzification methods to produce a complete FES.

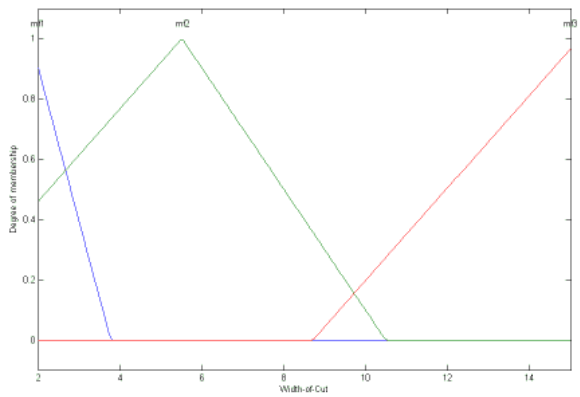

Figure 3.0 Membership functions from the FES

\section{B. Multi Objective Optimisation}

Since the pioneering work of Schaffer in 1985 [10] multiobjective genetic algorithms have been attracting increased interest. These algorithms seem particularly suited to this task. They process a set of solutions, the population, in parallel. This naturally allows them to generate a set of solutions approximating the pareto front. Schaffer developed the Vector Evaluated Genetic Algorithm (VEGA). This worked by creating sections or sub-populations in the next generation. Each subpopulation would select its parents according to one of the objectives. The whole population was then shuffled and crossover and mutation applied. This is known as a population based non-pareto approach.

Multi objective optimisation problems can prove very difficult. Objectives can be conflicting and incomparable. This prohibits the use of aggregation methods. In these cases a single optimum cannot be identified. Instead a set of non- dominated or efficient solutions are required. These are also known as the pareto optimal set. In minimisation problems the pareto set can be defined by: 


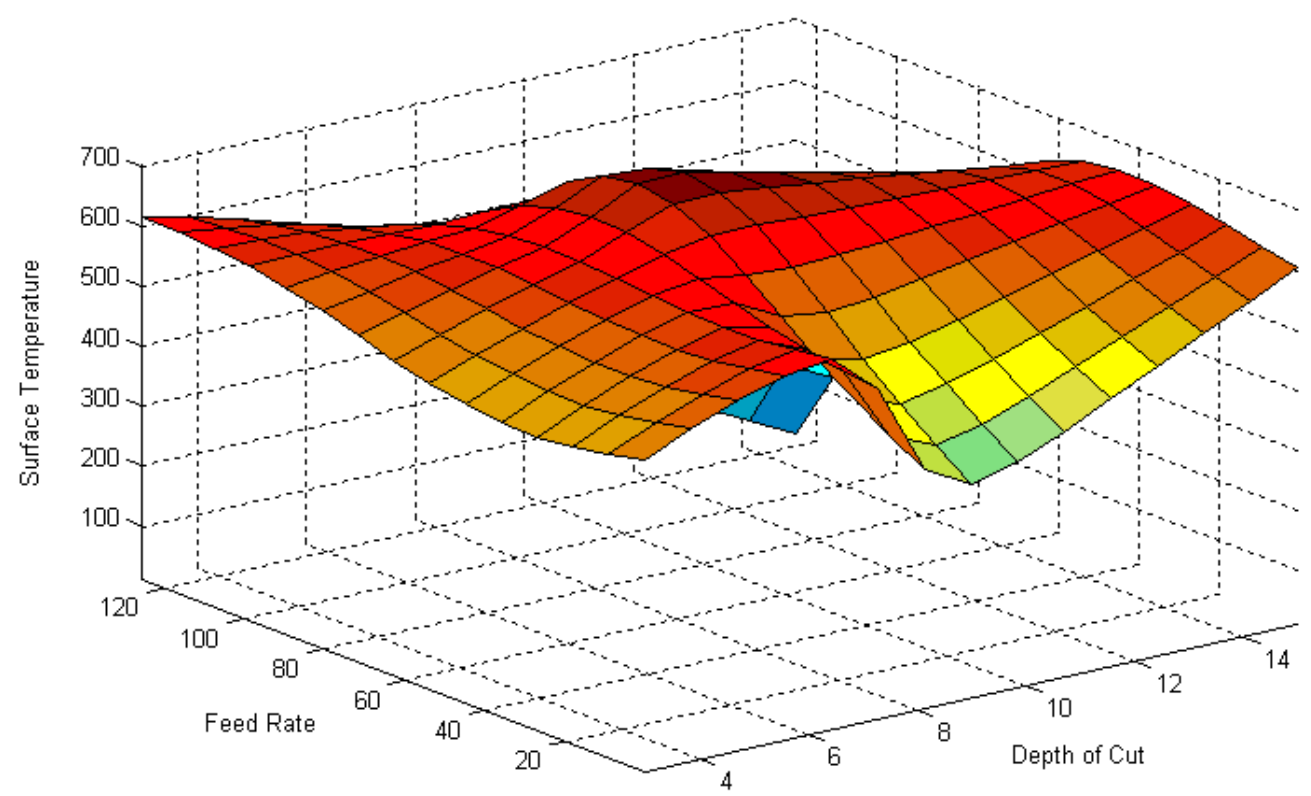

Figure 4.0 Surface Temperature

Definition 1 (Pareto Dominance) A vector $\mathrm{u}=\left\{\mathrm{u}_{1}, \mathrm{u}_{2}\right.$, $\left.\mathrm{u}_{3}, \ldots, \mathrm{u}_{\mathrm{p}}\right\}$ is said to dominate $\mathrm{v}=\left\{\mathrm{v}_{1}, \mathrm{v}_{2}, \mathrm{v}_{3}, \ldots, \mathrm{v}_{\mathrm{p}}\right\}$ if and only if $\mathrm{u}$ is partially less than $\mathrm{v} . \forall \mathrm{i}$ e $\{1,2, \ldots, \mathrm{p}\}, \mathrm{u}_{\mathrm{i}} \leq \mathrm{v}_{\mathrm{i}}$ and $\exists \mathrm{I}$ e $\{1,2, \ldots, \mathrm{p}\} \mathrm{u}_{\mathrm{i}}<\mathrm{v}_{\mathrm{i}}$.

Definition 2 (Pareto Optimality) A solution $\mathrm{x}_{\mathrm{u}} \mathrm{e} \mathrm{U}$ is said to be pareto optimal if and only if there exist no $\mathrm{xv}$ e $\mathrm{U}$ for which $\mathrm{v}=\mathrm{F}(\mathrm{xv})=\left\{\mathrm{v}_{1}, \mathrm{v}_{2}, \mathrm{v}_{3}, \ldots, \mathrm{v}_{4}\right\} \quad$ dominates $\mathrm{u}=\mathrm{f}\left(\mathrm{x}_{\mathrm{u}}\right)=\left\{\mathrm{u}_{1}, \mathrm{u}_{2}, \mathrm{u}_{3}, \ldots, \mathrm{u}_{\mathrm{p}}\right\}$.

Pareto based fitness assignment was first proposed by Goldberg [5]. The idea is to assign equal probability of reproduction to all non-dominated individuals in the population. It is also possible to rank the whole population according to how close it is to being nondominated and assign each rank a fitness value.

A similar scheme was proposed by Fonseca and Fleming [4], where each individual's fitness is calculated according to the number of individuals that dominate it. The more individuals that dominate a solution the lower that solution's fitness becomes.

More recently several new algorithms have been developed such as the Strength Pareto Evolutionary Algorithm (SPEA) [14] and Fast Elitist Non-dominated Sorting GA (NSGA-II) 21].

It has been observed that elitist GA's with pareto domination and diversity preserving operators achieve superior results[13].
In this study NSGA-II and SPEA algorithms will be applied to the problem.

\section{Proposed APPROACH}

The approach proposed in this paper is as follows. First the HEDG process is modelled using a fuzzy expert system. This model is then used as the fitness function in Multi objective evolutionary optimisation.

\section{A. Fuzzy Fitness Function}

A fuzzy expert system (FES) was created. The membership functions and rule base were developed using expert knowledge. A diagram showing the decision variables and objectives with respect to the FES is shown in figure 6.0. The knowledge was elicited through the use of structure interview techniques [3].

Empirical data was used to calibrate the model. The FES mapped the decision variables onto the objective function. It can be used as fitness function in an evolutionary algorithm.

The response surface for the surface temperature objective with respect to decision variables feed rate and depth of cut is shown in figure 4.0 


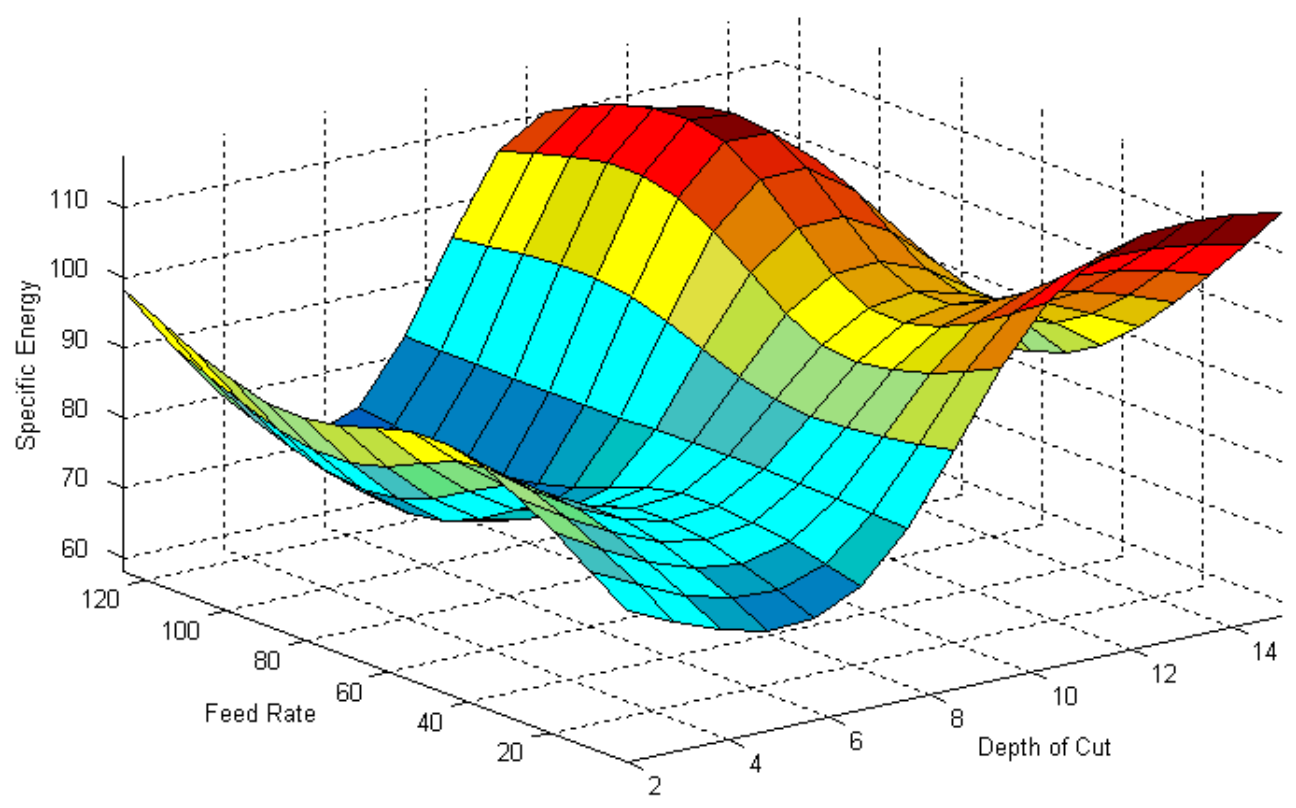

Figure 5.0 Specific Grinding Energy

Above, in figure 5.0, is the response surface for specific grinding energy with depth of cut and feed rate being the decision variables.

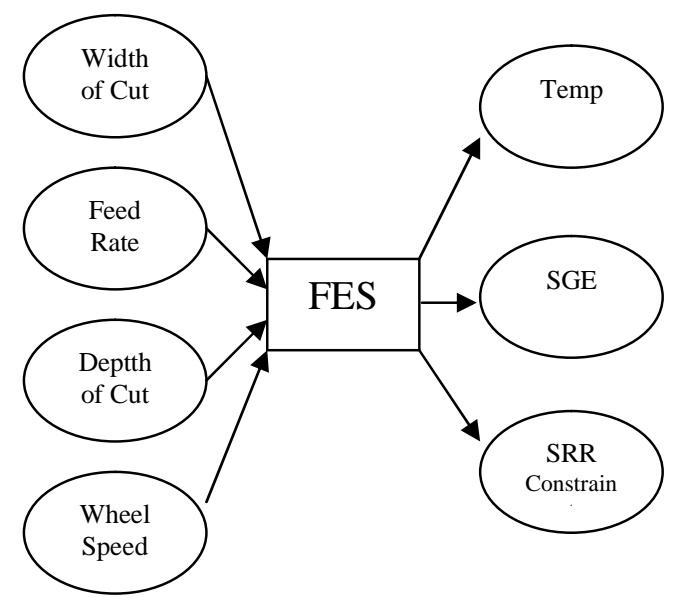

Figure 6.0 Schematic of fuzzy expert system

\section{B Constraints}

In Grinding the material is removed by the abrasive. It is then present in the gap or spaces between abrasives at the wheel's surface. The more gaps there are the greater the volume of space for chip removal. This parameter is known as the porosity of the wheel.

If the material removal per unit length of the wheel is higher than this porosity level material cannot be removed effectively. This constitutes a process constraint. In this study we shall model this constraint as a third objective. In this way infeasible solutions are valid during evolution. However feasible solutions will dominate infeasible solutions and thus few if any infeasible solutions will remain in the end population. This depends on how the objectives conflict. In a situation where several constraints are present they can be easily aggregated into one objective using a fuzzy expert system. This would represent the overall infeasibility of the solution.

In this study a value for material removed/wheel speed above 34 is infeasible. This was modelled using a fuzzy system. A membership function that has a degree of member ship equal to zero for values below 34 and constantly rises above this was used. A rule relating this to a consequent fuzzy set named infeasible was created. This set had a degree of membership value of zero at zero and grows proportionally greater as the value is increase. 


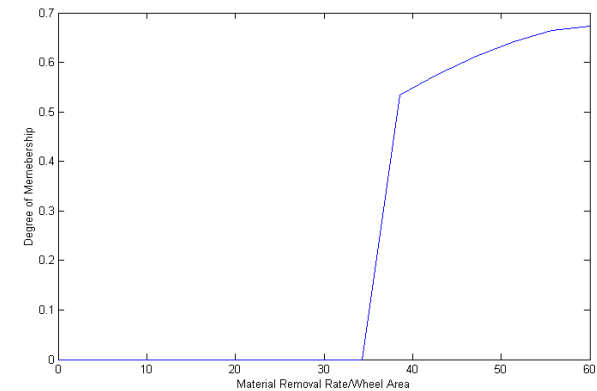

Figure 6.0 Output for fuzzy constraint objective

A plot showing the relationship between the constraint value and the degree of membership to the infeasible set is shown in figure 6.0. Extra constraints could easily be added. The fuzzy expert system allows the aggregation of several constraints to form one objective regarding the overall infeasibility of the solution.

When using floating point encoding is real numbers are recombined at a point $P$. The number $P$. is randomly generated in the interval $[0,1]$. For two real number $\mathrm{x}$ and $y$ the two offspring can be defined as

$\mathrm{x}^{\prime}=\mathrm{P}^{*} \mathrm{x}+(1-\mathrm{P}) \mathrm{y}$

$\mathrm{y}^{\prime}=\mathrm{P}^{*} \mathrm{y}+(1-\mathrm{P}) \mathrm{x}$

This means that new offspring formed by recombination always lie in between the parent values for each decision variable. Recombination in this manner is much more exploitative and less explorative when compared with binary crossover techniques. Real encoded GA's are totally reliant on mutation for exploration beyond current population boundaries.

It is hypothesised that allowing infeasible solutions to be included in the evolutionary process will give faster and repeatable convergence to the pareto front. Fuzzy constraints allow the inclusion of infeasible solutions.

\section{Search Space}

A quasi exhaustive search was carried out for this study to determine the nature of the search space. This is purely for academic reason and would not be necessary in industrial practice. It allows a qualitative evaluation of the nature of the search to be formed. It also allows the effectiveness of the MOEAs to be assessed. This plot only contains a fraction of the possible solutions. The plot contains $10^{4}$ points. The experiments were run using Matlab. Floating point numbers were to 15 significant figures. There are four decision variables hence total search space size of approximately $10^{(15+4)}$ points.

Figure 8.0 shows a scatter plot of the values for the two main objectives; surface temperature and specific grinding energy.
Infeasible solutions are included in this plot. Although difficult to see at this resolution figure 9.0 shows that the infeasible solutions can be detected near the pareto optimal front.

Figure 10.0 shows the SPEA algorithm run with the $3^{\text {rd }}$ objective measuring solution infeasibility included. The dark points represent feasible solutions. As the points fade they become more and more infeasible. This plot clearly shows that infeasible regions lie adjacent to the pareto front. Allowing infeasible solutions to be included in the evolutionary process gives a more rapid and reliable convergence to the pareto front.

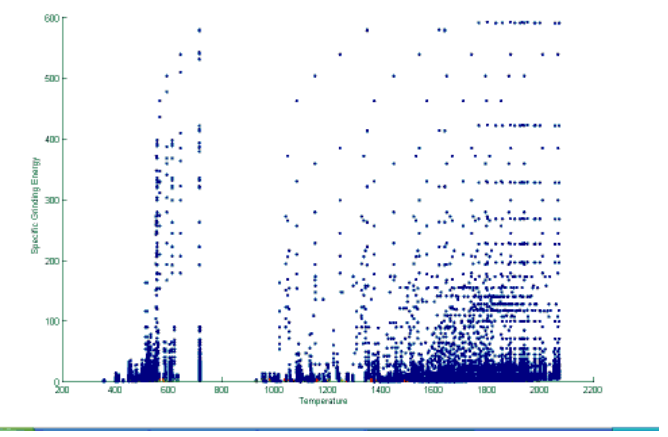

Figure 7.0 Quasi exhaustive search

\section{RESULTS FROM OPTIMISATION}

Two multi objective evolutionary algorithms (MOEA) were applied. NSGA and SPEA were chosen as two of the more highly regarded algorithms in the literature. The parameters set as shown in table 1.0.

Figure $11.0-15.0$ plot the results. The final population from the NSGA-II is represented by crosses. The archived set from the SPEA is represented by filled circles. Only feasible solutions are plotted.

NSGA produce a near optimum pareto front with good diversification in all cases. SPEA's results are inferior but still competitive except in the first case. With a limited number of generations, 10 in this case, SPEA finds points closer to the optimum point that NSGA-II.

SPEA only retains non dominated solutions in the archive. It is in effect much more selective about which candidates it favours. This effect is only apparent before the archive reaches its maximum. The SPEA quickly approximates the pareto front but does not retain diversity as effectively. Instead it relies on the fitness assignment and crowding factor to diversify.

The NSGA algorithm is slower at converging towards the front but given time produces a more diverse estimate that is closer to the real front. 

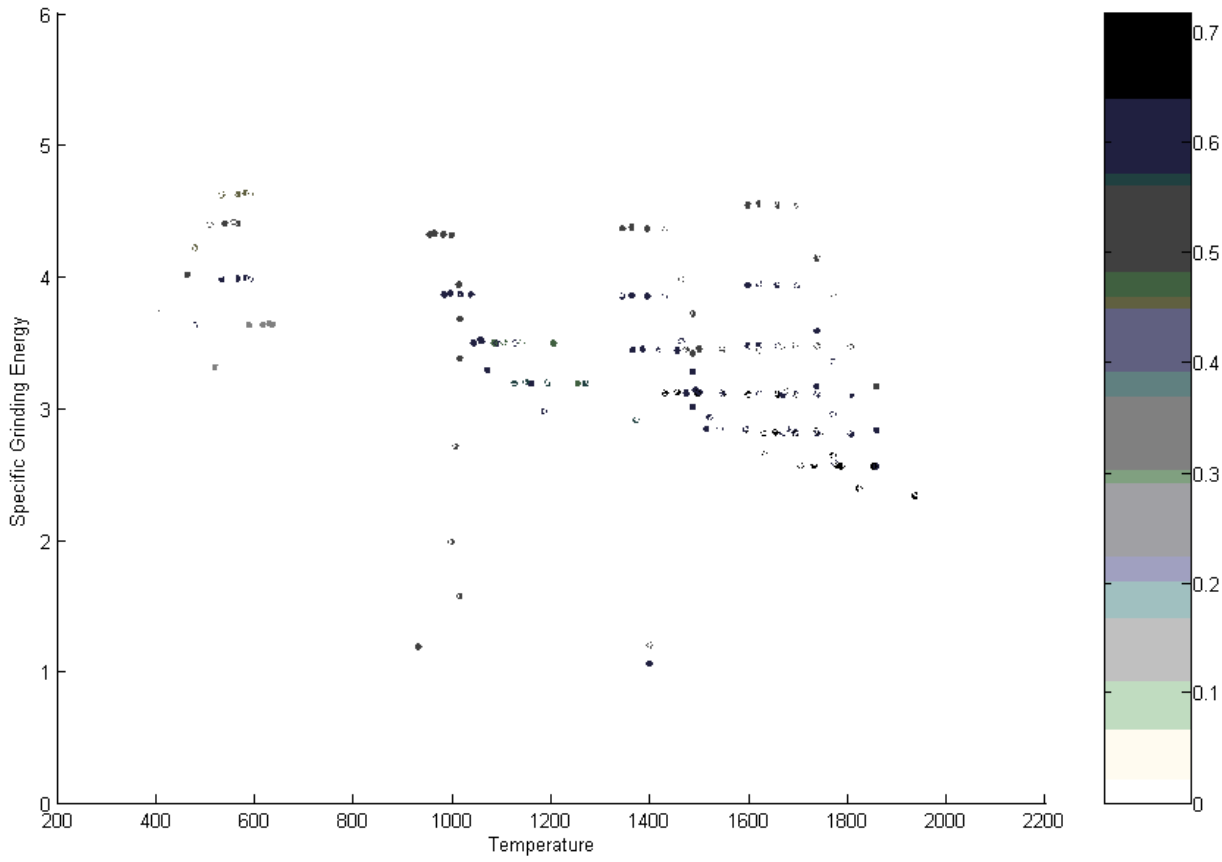

Figure 8.0 Infeasible values close to pareto front

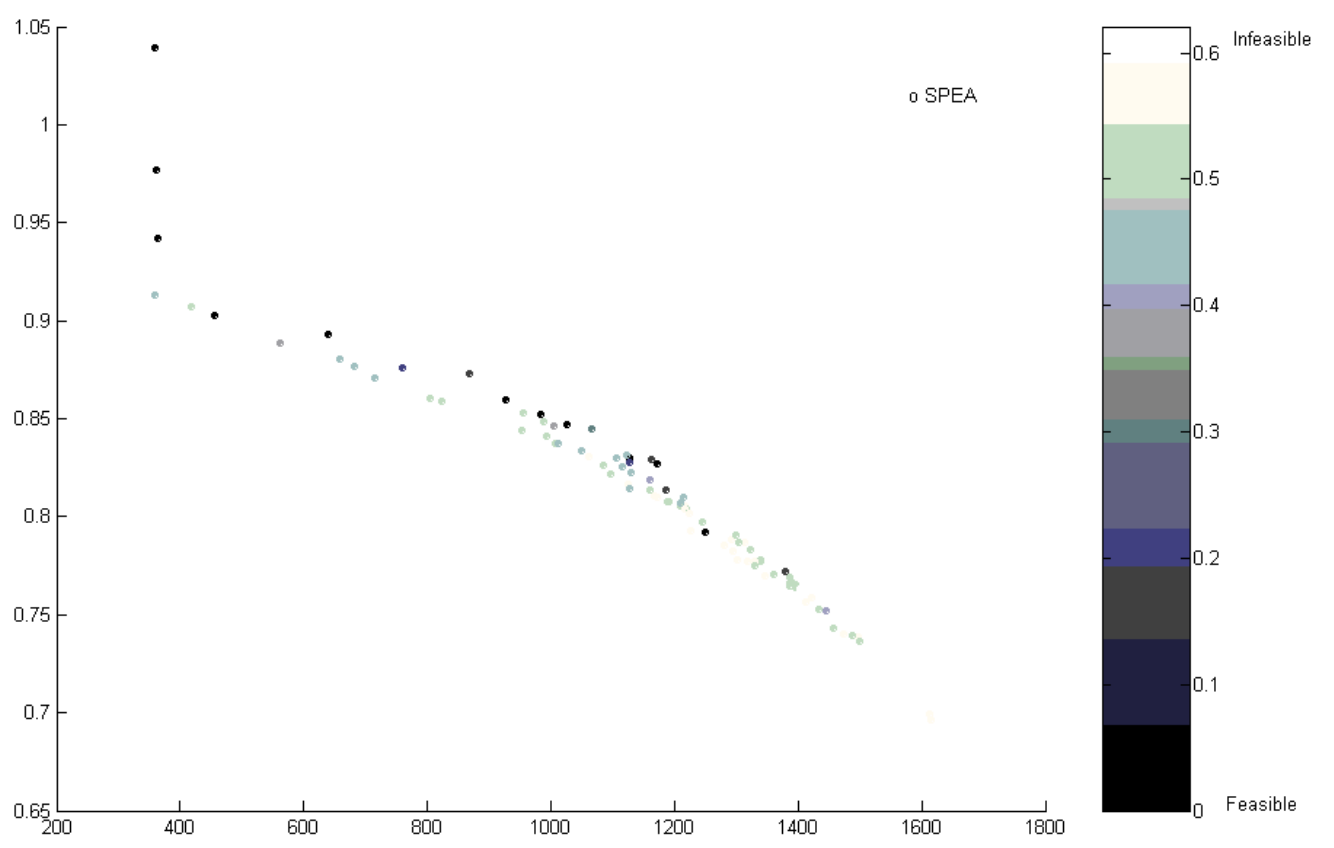

Figure 9.0 SPEA using solution feasibility as third objective 


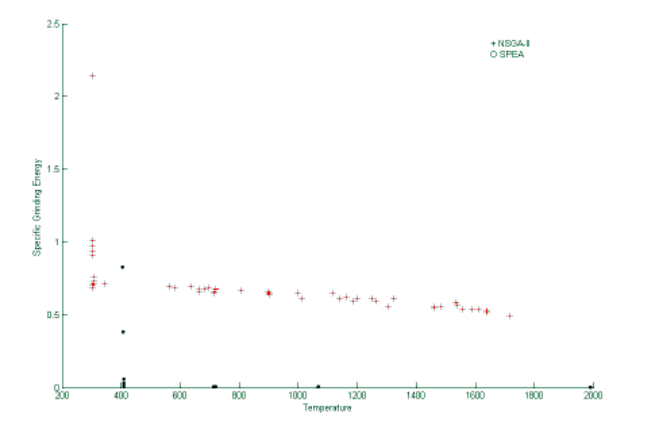

Figure 10.0 NSGA and SPEA pareto front estimatations

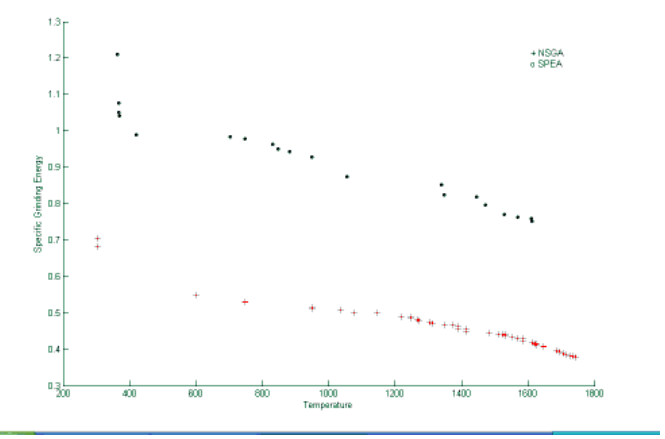

Figure 11.0 NSGA and SPEA pareto front estimatations

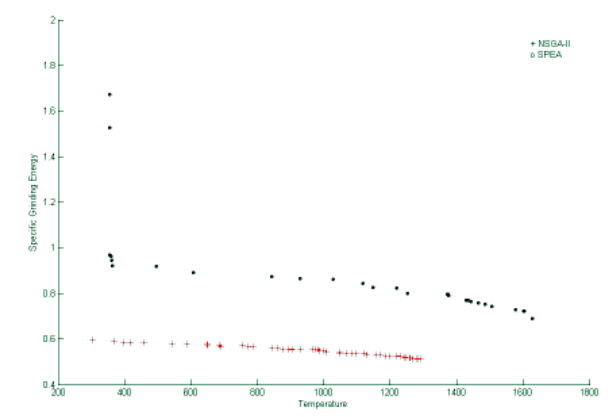

Figure 12.0 NSGA and SPEA pareto front estimatations

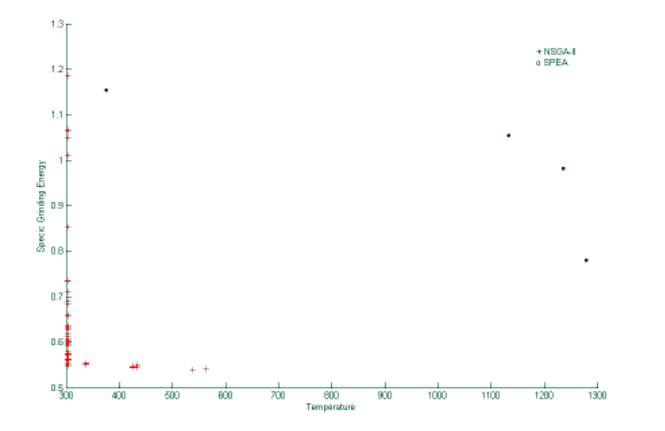

Figure 13.0 NSGA and SPEA pareto front estimatations

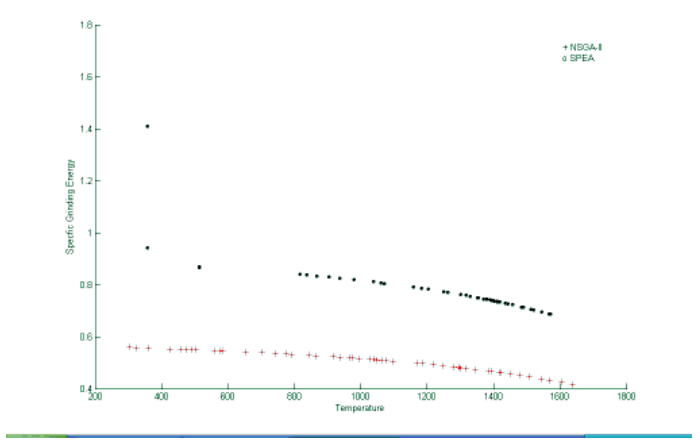

Figure 14.0 NSGA and SPEA pareto front estimatations

\begin{tabular}{|c|c|c|c|c|c|}
\hline Fig & Pop & Arch & Xover & Mutate & $\begin{array}{c}\text { Genera } \\
\text { tion }\end{array}$ \\
\hline 1 & 50 & 50 & 0.8 & 0.2 & 10 \\
\hline & 50 & - & 0.8 & 0.2 & 10 \\
\hline 2 & 50 & 50 & 0.8 & 0.2 & 30 \\
\hline & 50 & - & 0.8 & 0.2 & 30 \\
\hline 4 & 50 & 50 & 0.8 & 0.2 & 100 \\
\hline & 50 & - & 0.8 & 0.2 & 100 \\
\hline 4 & 50 & 50 & 0.8 & 0.4 & 30 \\
\hline & 50 & - & 0.8 & 0.4 & 30 \\
\hline 5 & 50 & 50 & 0.5 & 0.2 & 30 \\
\hline & 50 & - & 0.5 & 0.2 & 30 \\
\hline
\end{tabular}

Table 1.0 Parameters for algorithm experiments 


\section{Discussion}

In many cases MOEA can effectively estimate the pareto front with a high proximity. They can often find it difficult to converge exactly onto the front. One reason for this could be the use of real encoding when the solution lies on one or more boundary constraints. If infeasible solutions are not allowed in the evolutionary process a real encoded GA is relying on either a member of the initial population or a mutation to create an individual on this pareto front.

The use of fuzzy constraints as an objective allows infeasible solutions to take part in the evolution of the pareto front. This increases the likelihood of the pareto front lying between points included in the initial population.

The experiments showed how NSGA outperforms SPEA under all conditions when applied to this problem. The only exception is with a low number of generations. Here SPEA convergence to the pareto front is quick whilst its diversity is inferior

\section{LIMITATIONS AND FUTURE RESEARCH}

This paper has shown that using fuzzy expert systems as fitness functions in evolutionary optimisation is a valid and effective approach. It also shows that modelling constraints as part of the fuzzy system is possible. Research into the advantages of using this technique compared to alternatives is needed.

Typically a fuzzy model take longer than a mathematical equation to evaluate. In the literature, when comparing multi objective algorithms the number of sorts required has been used to evaluate an algorithm's complexity and thus the time required for operation. When using fuzzy models, or indeed any fitness function with a relatively long evaluation time the number of sorts becomes less important. The number of evaluations is significant. Research into a MOEA specifically aimed at operating with long fitness function evalution times is needed. This would put less emphasis on archive size and number of sort and more on number of evaluations. This could come in the form of an algorithm that combines memory methods (tabu search etc) with evolutionary methods. More research is needed in this area.

SPEA outperforms NSGA for the first few generations. Research is required to determine if this is the case ifor the majority of problems. If so can an algorithm be developed that combines the strong points of each method.

\section{CONCLUSIONS}

This paper has applied fuzzy based modelling techniques to the HEDG process and created a meaningful model. It has also shown how constraints can be modelled as fuzzy objectives. The greater the degree to which a solution breaks constraints the higher its degree of membership to the set 'infeasible' becomes. The paper has successfully applied two of the leading MOEA to the problems. This proves that fuzzy modelling, and evolutionary algorithms are effect techniques in the optimisation of industrial processes.

More research is needed in this area. In particular a comparison of MOEA using fuzzy constraints against other constraint modelling techniques is required.

\section{REFERENCES}

1. Bhuvaneshwaran, V. and Langari, R. (2003) Design and Optimisation using Genetic Algorithms and Fuzzy Constraints and Fitness Functions. Transactions of the IEEE International Conference on Fuzzy Systems. pages 354-359.

2. Deb K., Agrawal, S., Pratab, A. and Meyarivan, T. (2000) A fast elitist non-dominated sorting genetic algorithm for multiobjective optimization: NSGA-H, KanGAL Technical Report 200001, Indian Institute of Technology, Kanpur, India.

3. Durkin J. (1994) Expert Systems, Design and Development. Prentice Hall International.

4. Fonseca, C.M. and Fleming, P.J.(1993). Genetic Algorithms for Multi-objective optimization: Formulation, discussion and generalization. In Genetic Algorithms: Proceedings of the Fifth International Conference. Morgan Kaufmann, San Mateo, CA, pages 416-423.

5. Goldberg, D.E., (1989). Genetic Algorithms in Search Optimisation and Machine Learning. Addison-Wesley.

6. T. Jin, D. J. Stephenson and J. Corbett, Burn threshold of high carbon steel in high efficiency deep grinding, 2002, Proc. Institution of Mechanical Engineers, Part B, J of Engineering Manufacture, Vol. 216, 357-364

7. T Jin and D J Stephenson, 2002, New developments of High efficiency deep grinding, Abrasives Magazine, April/May issue, pp.1-4

8. Michalewicz, Z.(1996).: Genetic Algorithms + Data Structures = Evolution Programs. 3rd edn. SpringerVerlag.

9. W Brian Rowe, Tan Jin, 2001, Temperatures in high efficiency deep grinding (HEDG), Annals of CIRP, Vol. 50/ 1, 205-208

10. Schaffer, J.D. (1985). Multiple objective optimization with vector evaluated genetic algorithm. In: Genetic algorithms and their applications: Proceedings of the first international conference on genetic algorithms, 93-100, Lawrence Erlbaum, USA.

11. D. J. Stephenson and T. Jin, 2003, Physical basics of grinding, The first European Conference of Grinding, Aachen, November, Germany

12. Tawakoli, T. (1990). High Efficiency Deep Grinding. VDI-Verlag GmbH, dusseldorf, Germany.

13. Tiwari, A. (2001). Development of Flexible Optimisation Techniques for Wengineering Design. PhD. Thesis, department of Enterprise Integration, Cranfield University, Bedford, UK.

14. Zitler, E. and Thiele, L. (1998). An evolutionary algorithm for multiobjective optimization: The strength Pareto approach. Technical Report No. 43, Computer Engineering and Networks Laboratory, Zurich, Switzerland. 